Cita bibliográfica: Cazorla Palomo, J. y Parra Ramajo, B. (2017). El cambio en los modelos del trabajo social en salud mental: del modelo rehabilitador al modelo social [Changes on social work models in mental health: from the rehabilitation model to the social model]. Alternativas. Cuadernos de Trabajo Social, 24, 43-54. https://doi.org/10.14198/ ALTERN2017.24.03

\title{
EL CAMBIO EN LOS MODELOS DEL TRABAJO SOCIAL EN SALUD MENTAL: DEL MODELO REHABILITADOR AL MODELO SOCIAL
}

\section{CHANGES ON SOCIAL WORK MODELS IN MENTAL HEALTH: FROM THE REHABILITATION MODEL TO THE SOCIAL MODEL}

\author{
Josep CAZORLa PALOMO \\ Universitat de Barcelona, Facultat d'Educació, Escola de treball social, Barcelona, España \\ josepcazorla@ub.edu \\ BELÉN PARRA RAMAJO \\ Universitat de Barcelona, Facultat d'Educació, Escola de treball social, Barcelona, España \\ belenparra@ub.edu
}

\section{Resumen}

El modelo rehabilitador es en la actualidad el modelo rector de nuestra sociedad y del mundo de la atención de la Salud Mental. Este estándar exige al individuo que se adapte al medio, responsabilizándolo de sus limitaciones y rechazando la diferencia. Desde esta visión se transforma a la persona en un usuario o un consumidor de servicios, reforzándose la discriminación a partir de los ideales establecidos y los mecanismos de control institucionales.

El trabajo social emerge, desde sus inicios, como una disciplina que reconoce las capacidades y las experiencias de las personas de forma singularizada, para facilitar que sean estas las protagonistas de sus relatos de vida. Es por ello, que en el ámbito de la Salud Mental uno de los principales propósitos es la descategorización de la diferencia que perpetua el estigma. Esta concepción se encuentra estrechamente unida al modelo social, perspectiva que concibe toda vida humana con las mismas condiciones de dignidad que el resto de la sociedad, que facilita a las personas con trastornos mentales el reconocimiento como ciudadanos con plenos derechos y deberes dentro de la sociedad en la que habitan.

Palabras clave: Trabajo Social, Salud Mental, Diversidad, Participación, Estigma, Modelo Rehabilitador y Modelo Social.

\begin{abstract}
Currently, the rehabilitation approach is the guiding model in mental health care as well as in society in general. This standard demands individuals to fit in their environment, making them responsible for their limitations and rejecting difference. From that point of view, a person becomes a user or a consumer, which reinforces discrimination through established ideas and mechanisms of institutional control.

From the beginning, social work emerges as a discipline that recognizes individuals' capacities and experiences in a singular way, making it easier for them to star in their life story. For that reason, one of the main goals in mental health care is the decategorization of the difference which perpetuates the stigma. This idea is closely linked to the social approach, which conceives the whole human life in terms of equal dignity conditions, which makes it easier for people with mental disorders to be acknowledged as citizens with full rights and obligations in the society they are living
\end{abstract} in.

Keywords: social work, mental health, diversity, participation, stigma, rehabilitation approach, and social approach. 


\section{EXTENDED ABSTRACT}

Currently, the rehabilitation approach is the guiding model in mental health care as well as in society in general. This standard demands individuals to fit in their environment, making them responsible for their limitations and rejecting difference. From that point of view, a person becomes a user or a consumer, which reinforces discrimination through established ideas and mechanisms of institutional control.

From the beginning, social work emerges as a discipline that recognizes individuals' capacities and experiences in a singular way, making easier for them to star in their life story. For that reason, one of the main goals in mental health care is the decategorization of the difference which perpetuates the stigma. This idea is linked to the social model, which conceives the whole human life in terms of equal dignity conditions, which makes it easier for people with mental disorders to be acknowledged as citizens with full rights and obligations in the society they are living in.

People who suffer from mental disorders have to face numerous challenges. The diagnosis of a mental disorder triggers a succession of consequences that in result will hugely impact and transform the lives of those who are diagnosed as they will endure high quotes of discrimination, stigmatization and psychosocial distress. The biggest challenge they have to face is the fact that they have been linked to categorizations considered mental disorders, also called stigma. Every time that stigma is studied in diagnosed individuals, feelings of shame and being blamed by others appear at the centre of their perception. The damaging effects of stigma spread over all aspects of life of those diagnosed with a mental disorder making it more difficult for them to get a job or housing, for instance, damaging also their self-esteem as a consequence of the internalized stigma.

It is for that reason that, from mental health care, the decategorization of the difference that perpetuates the stigma is one of the main functions of social work. That is why it is important to become aware of the effects of the diagnosis over the professional relationship, in order to preserve singularity, highlighting and counteracting the ways in which stigma is expressed. Social work in mental health care has to be focused on fighting stigma, and on improving the wellbeing and the «social place» through a sense of belonging and self-capability in people diagnosed with mental disorders, working with them to maximize their strengths and minimize social processes that undermine their social identities.

For a long time, the biomedical model has been the main assistance model in mental health care and in social work. This model is based on focusing on the deficit and on a causal understanding of mental disorders, and on the sense of a chronic course of the mental pathology. This perspective focuses its interest on the cure or treatment of the difference, using policies and interventions with the purpose of normalizing the people diagnosed with mental disorders, making them responsible for the difficulties in adapting to the environment they are living in.

Several voices have warned about the incorporation of the biomedical model by social workers, as it has been not thought through and implemented without criteria; a model that also in social work emphasizes the linear process of the intervention, through which the clinical symptoms that lead to a diagnosis are identified in order to provide treatment, help or intervention guiding. As a consequence, the recovery model emerges as a perspective that causes a philosophical and conceptual change, through changing illness and deterioration as attention cores for strengths and capacities. The recovery model is based on the belief that despite the traumatic experiences linked to a mental health diagnosis, it is possible to rebuild a satisfactory and significant life. This philosophy highlights that recovery is about living a hopeful, significant and purposeful life, and it is equivalent to reaching the capacity for transcending symptoms, functional incapacity and social limitations that influence the disorder. It is about recovering the roles as individuals and citizens.

If we examine the recovery model in depth, we will find that the values and principles on which it is based are the same central values of every social work practice. In the recovery model other elements of the social work practice can also be found, such as listening to people, accepting them just the way they are, and trusting their potential. Another confluent element between social work practice and the recovery perspective is participation. The professional social work practice is performed from the principle that it conceives that every individual is able to identify and deal with their own difficulties, an affirmation which is supported by their strengths, capacities, and abilities. Participation facilitates the change of values and attitudes from society, because by competently voicing and sharing their experiences their knowledge is recognized (Corrigan et al., 2001).

In spite of the recovery model's goodwill, there are forms of discrimination and inequality that persist in its basis and that the implementation of the model could not avoid, because in order to measure and range disability and the access to resources, classifications are still being used. This contributes again to a new control mechanism to resources access and subsequently discrimination against those unable to develop a life project within the established ideas (Mulvale y Bartram, 2015). This paradox has favoured the appearance of a new and more critical position on the discipline, which resulted in the introduction of the social model.

The social model, also called mental diversity model, highlights a change in attitude and a political change within the community towards people's limitations and barriers. This philosophical line of thought postulates that everyone has many characteristics that make them unique beings, asking the community to understand that they should neither protect nor separate from these individuals because they are one of us and, therefore, valuable members of the whole humanity.

The social model of mental diversity makes an appeal to social workers to implement a qualitative and political change in the responsibility for the barriers imposed to people diagnosed with a mental disorder. This change may contribute to the recognition of people's experience as an essential element for the understanding of the situation, and for its approach as well (Brea y Gil, 2016). This change entails a professional challenge because it involves the change on the establishment of the role accepted by the person as a service user to the role of expert and ally towards overcoming the biomedical model in mental health care. With this purpose, social workers must facilitate inclusion of diversity in society through relational nets and community work, to promote the improvement in quality of life of those diagnosed with a mental disorder, through palliating the stigma and inequalities that could emerge (Muyor, 2011).

The acceptance of the social model facilitates those who suffer from a mental disorder are recognized as citizens with full rights and duties within the society they live in.

Keywords: social work, mental health, diversity, participation, stigma, recovery model and social model. 


\section{Introducción}

Los principios y valores del trabajo social enuncian el reconocimiento de la diversidad y la participación activa de las personas en los procesos de intervención, como ejes fundamentales que facilitan el cambio. Para ello se propicia el protagonismo de la persona durante todo el proceso, para así alcanzar el control sobre su propia historia y la mejora de la calidad de vida. Para materializar dichos valores es fundamental que los/las trabajadores/as sociales, desde una mirada crítica, sean capaces de romper con las categorizaciones totalizadoras establecidas socialmente, en pro del reconocimiento de la singularidad del individuo como sujeto único (Derrida, 1997). Porque la reducción de las personas a una idea o prejuicio preconcebidos, perpetua la desigualdad mediante el control y la dominación de la diferencia (Idareta, 2013), reduciendo la capacidad de obrar, y el ejercicio de los derechos y deberes como ciudadanos activos, de las personas diagnosticadas con un trastorno mental.

Con el mismo objetivo que conduce a la plena participación, los/las trabajadores/as sociales reconocen las capacidades y la experiencia de aquellas personas que han sido ignoradas y silenciadas por la sociedad y por las organizaciones que ejercen el poder. Es por ello que la relación profesional que se establecerá será simétrica, mediante dicha relación, el/la profesional conocerá a la persona de forma integral, comprendiendo todos los aspectos de su vida, evitando que se conciba como una simple tarea a la que dar una solución (Farah, Ward, Woolner y Tumer, 2011).

Para ello es necesario que los principios éticos en los que se promueven la dignidad, los derechos, el respeto, la privacidad y la confidencialidad de las personas (Banks, 1997), rijan la intervención, en contraposición a la marginación y al aislamiento individual y social, la privación de derechos y la desigualdad, porque todo ello provoca la aparición de sentimientos de miedo, culpa y soledad (Thornicroft, 2007).

Desde esta perspectiva, la práctica de la disciplina se aproxima a las personas diagnosticadas con un trastorno mental en su vertiente individual y emocional, trabaja con ellas y con sus familias para asegurar que sus derechos ciudadanos no sean vulnerados, y para garantizar su acceso a los recursos en situación de igualdad; ofreciendo una relación profesional que les posibilite hacer frente a las consecuencias de la exclusión y la desigualdad (Cazorla, 2014). Requiere también de una necesaria participación en la comunidad que conecte el malestar individual con una conciencia crítica que concrete cambios en el contexto social (Parra; Oriol y Martínez, 2011)

Los/las trabajadores/as sociales que desarrollan su práctica en el ámbito de la salud mental conciben y despliegan las estrategias que contribuyen a 
mejorar el bienestar y el «lugar social» mediante el fomento de la pertenencia y el sentido de capacidad de las personas diagnosticadas con un trastorno mental, trabajan con las personas para maximizar sus fortalezas y minimizar los procesos sociales que devalúan su identidad social.

\section{El impacto del diagnóstico}

Los diagnósticos de trastorno mental desencadenan una serie de consecuencias de gran impacto que transforman las vidas de las personas que los reciben, llegando a padecer elevadas cuotas de discriminación, desigualdad, estigmatización y sufrimiento psicosocial. Las personas con trastornos mentales tienen que hacer frente a múltiples retos, a la recuperación del significado de la vida, a los sentimientos depresivos, a las ideas suicidas, la vergüenza, la pérdida de la capacidad para disfrutar de la vida, la alienación social y el estigma internalizado (Gelkopf, y Roe, 2014; Michaels, López, Rüsch y Corrigan, 2012).

Diversas investigaciones que analizan en profundidad las experiencias de vida de las personas diagnosticadas de trastorno mental coinciden en destacar que en las experiencias cotidianas de estas personas, se incrustaran vivencias de profundo dolor, aislamiento e impotencia, de injusticia y de derrota. Estas experiencias de sufrimiento aumentan, con frecuencia, por la atención recibida desde servicios, o bien coercitivos o paternalistas (Tew, Shul, Slade, Bird, Melton y Le Boutillier, 2012).

Igualmente en un amplio estudio realizado sobre la percepción de las personas con un diagnóstico grave de salud mental, de la desesperación y el estigma internalizado, se muestra la incidencia negativa del diagnóstico en las experiencias personales y sociales (Williams, Almeida y Knyahnytska,2015).

El mayor reto que tienen que superar las personas inscritas en las clasificaciones diagnosticas que enuncian trastornos graves de salud mental es el estigma, entendido este como todas aquellas características indeseables que se asocian a las enfermedades mentales, que se colocan en categorías que identifican la diferencia entre aquellos que las presentan y los que no (Buck, 2013).

Siempre que se investiga sobre el estigma, en la percepción de las personas diagnosticadas, aparecen en el centro las experiencias de vergüenza, y la vivencia de ser culpabilizadas por los demás. Los efectos nocivos del estigma se extienden en todas las dimensiones de la vida de las personas con trastornos mentales, dificultando la obtención de trabajo o de vivienda por ejemplo, dañando también la autoestima como consecuencia de la internalización del estigma. En comparación con otras circunstancias altamente estigmatizadoras, la enfermedad mental es la más estigmatizante, es por ello que se suele denominar «el estigma extremo» (Thornicroft, 2007). 
Como afirma Idareta (2014), los procesos de categorización de problemas y grupos de población se realizan para poder comparar, medir y calcular, pero «tras la categorización, se debe proceder a descategorizar a cada sujeto. Con la categorización se eliminan todas aquellas cualidades que hacen al sujeto singular, sustituyéndolo por una idea que uniformiza a todas ellas» (Idareta, 2014, pp.17-18).Es por ello que las y los trabajadores sociales deben tomar conciencia de los efectos de la utilización del diagnóstico en la relación profesional, para salvaguardar la singularidad y señalar y contrarrestar las formas en que se expresa el estigma (Michaels, López, Rüsch, y Corrigan, 2012).

Los modelos de atención a la salud mental y la práctica del trabajo social. El modelo médico, el modelo de la recuperación y el modelo social

Durante largo tiempo el modelo imperante de atención en la salud mental, y por ende en el trabajo social en salud mental, ha sido el denominado modelo médico. Este se centra en el déficit, y pone el énfasis en una comprensión causal de los trastornos y en el sentido de un curso crónico de la patología mental (Mulvale y Bartram, 2015). El modelo médico o rehabilitador surgió a principios del siglo XX como evolución de su predecesor, el modelo presidencialista, en el que se entendía la diversidad como un castigo divino, para así transformar esta concepción en enfermedades a las cuales no eran necesarias marginar de la sociedad (Palacios y Romañach, 2006). Desde este enfoque se responsabiliza al individuo de las dificultades de la adaptación al medio en el que habita, centrando su interés en la cura o el tratamiento de la diferencia (Victoria, 2013). Así, desde esta concepción, se pone de relieve la diferencia, estableciéndose políticas y actuaciones que tiene como finalidad la normalización de la persona que sufre una enfermedad mental (Asís, 2013), considerándose imprescindible que este colectivo sea rehabilitado como acción necesaria para su reincorporación como parte de la sociedad de pertenencia (Velarde, 2012).

Puesto que la atención centra su interés en la ausencia de salud, esta se torna en un rasgo que infravalora las actitudes de las personas en relación al resto de la sociedad, propiciando una atención social basada frecuentemente en el paternalismo (Toboso y Arnau, 2008). Desde este modelo la atención es eminentemente jerárquica, el profesional es el conocedor de la solución, prioriza la rehabilitación del individuo, para de esta forma fomentar la creación de una identidad como enfermo (Velarde, 2012) sin tener en cuenta la voluntad de la persona, al ser considerada como menos valorada que el resto (Palacios y Romañach, 2006).

Diversos autores han alertado sobre la incorporación no reflexionada y acrítica del modelo médico por parte de los/las trabajadores/as sociales, en los 
últimos tiempos (Beecher, 2009; Bradhaw, Armour y Roseborough, 2007; Hyde, Bowles, y Pawar, 2015; Karban, 2017), modelo que también en el trabajo social enfatiza un proceso lineal de intervención mediante el cual se identifican los síntomas clínicos que conducen a un diagnóstico para proporcionar el tratamiento, la ayuda o guiar la intervención.

Como consecuencia surge el modelo de la recuperación, perspectiva que provoca no solo un cambio filosófico sino también conceptual al cambiar la enfermedad y el deterioro como núcleo de atención, imperante en el modelo biomédico, por las fortalezas y las capacidades. El modelo de la recuperación afirma que a pesar de las experiencias traumáticas asociadas a un diagnóstico de salud mental, se pueden reconstruir vidas satisfactorias y significativas.

La recuperación en salud mental se centra en tres áreas interconectadas: a) potenciación y control sobre la propia vida, b) inclusión social, relaciones interpersonales y reconstrucción de identidades positivas en contra de la discriminación y el estigma, c) esperanza sobre el futuro y encontrar un sentido y un propósito de vida (Tew et al, 2012).

En definitiva, tal y como plantean Williams, Almeida y Knyahnytska (2015), la recuperación consiste en vivir una vida con esperanza, significado y propósito. O como matizan Bergeron-Leclerc y Cormier (2009), la recuperación equivale a lograr la capacidad para transcender los síntomas, la incapacidad funcional y las limitaciones sociales que influyen sobre el trastorno; consiste en la recuperación del rol de sujeto y ciudadano. En la misma línea, importantes estudios como el de Williams, Almeida y Knyahnytska (2015), enuncian que los elementos fundamentales para lograr la recuperación son la sensación de capacitación y el derecho a la participación plena en el entorno social. Estos autores instan al trabajo social en el ámbito de la salud mental a dirigir sus objetivos a la promoción de la plena ciudadanía de las personas diagnosticadas, a trabajar para combatir el estigma. En consonancia, Buck (2013) también señala que una de las principales finalidades del trabajo social en salud mental es la creación de contextos sociales donde las personas diagnosticadas con trastornos mentales puedan encontrar sentido y esperanza después de haber recibido el diagnóstico.

Algunos autores como Bergeron-Leclerc, y Cormier (2009) y Carter, Pagliano, Francis y Thorner (2016) sustentan que los valores y principios del modelo de la recuperación son los mismos que los valores centrales de toda práctica del trabajo social. Enuncian que las intervenciones de trabajo social preservan elementos definitorios que también son propios, y así han sido enunciados, como constituyentes del modelo de la recuperación. Concretamente, elementos tales como la escucha, la aceptación de la persona tal como es, 
la creencia en su potencial, se identifican como factores que contribuyen al éxito de la intervención en el campo de la salud mental (Bergeron-Leclerc y Cormier 2009). Otro de los elementos señalados, confluente entre la práctica del trabajo social y la perspectiva de la recuperación es la participación. La práctica profesional del trabajo social se realiza desde el principio mediante el que concibe a todo individuo como capaz para identificar y abordar sus propias dificultades, afirmación apoyada en las fortalezas, las capacidades y las habilidades de la persona. La participación posibilita el cambio de actitudes y de valores en la sociedad, puesto que al dar la voz con competencia, y compartir experiencias se reconoce el conocimiento de todas las personas (Corrigan et al., 2001). De forma similar, la literatura sobre el modelo de la recuperación resalta que el reconocimiento de toda experiencia particular facilita el camino hacia la recuperación, mediante la participación de las personas diagnosticadas con un trastorno mental en sus procesos, como expertos y como fuente de conocimiento, hecho que fomenta la autonomía y genera el sentimiento de dignidad (Bradhaw, Armour y Roseborough, 2007).

Sin embargo, a pesar de la buena voluntad que acoge el modelo de la recuperación, en sus fundamentos persisten formas de discriminación y desigualdad que la aplicación del modelo no ha podido evitar, ya que se continua utilizando clasificaciones para medir y graduar la discapacidad y el acceso a los recursos, cuestión que de nuevo genera mecanismos de control y acceso a los recursos, y por lo tanto de discriminación de las personas que no hayan podido desarrollar un proyecto vital que siga los ideales marcados (Mulvale y Bartram, 2015). Esta paradoja ha facilitado la emergencia de posturas más críticas en la disciplina, que han dado lugar a la emergencia del denominado modelo social, que se exporta del movimiento basado en la vida independiente, nacido en Estados Unidos en el campo de la diversidad funcional (Aullé, 2003), que concibe toda vida humana con las mismas condiciones de dignidad que el resto. El modelo social, llamado también de la diversidad mental, apunta a un cambio actitudinal y político de las comunidades hacia las limitaciones y barreras de las personas. Desde esta perspectiva filosófica se postula que todas las personas poseen una serie de características que las convierten en seres únicos, interpelando a la comunidad a entender que no es necesario protegerse o apartarse de ese individuo, ya que es uno más de nosotros y, por tanto, una parte del conjunto valioso de la humanidad.

Por ello, tal y como enuncia Sen (1988), no es concebible un único tipo de comprensión del mundo y de las personas, ya que en la misma idea de igualdad existe la desigualdad, por lo que se prima la elección individual de cada persona a ser y a poder escoger ser diferente, y que esta libertad de elección se 
erija en derechos que puedan ser atendidos. Desde este pensamiento, el trabajo social se responsabiliza de la aceptación de la diferencia, lo que propiciará la participación dignificada de la persona diagnosticada con un trastorno mental dentro de la sociedad como un ciudadano más.

\section{Retos en la práctica del trabajo social}

El modelo social de la diversidad mental invoca a los/las profesionales del trabajo social a un cambio cualitativo y político en relación a la responsabilidad sobre las barreras que se imponen a las personas diagnosticadas con un trastorno mental. Este giro propiciará el reconocimiento de la experiencia de las personas como elemento imprescindible para la comprensión y el conocimiento de la situación, así como para su abordaje (Brea y Gil, 2016).El cambio supone un reto profesional ya que implica que se modifique el hasta ahora asumido papel de la persona como usuaria del servicio, por el de experto y aliado, superando el modelo biomédico de la salud mental. Es por ello que en su práctica, los/las trabajadores/as sociales han de favorecer la inclusión de la diversidad en la sociedad mediante las redes relacionales y el trabajo comunitario, para propiciar una mejora en la calidad de vida de las personas diagnosticadas con un trastorno mental, mediante la paliación de los estigmas y las desigualdades que se pueden generar (Muyor, 2011).

La creación y/o el mantenimiento de relaciones sociales propician en los sujetos la capacidad de asumir su protagonismo social, de dignificar sus relatos vivenciales y de crear nuevas perspectivas relacionales más allá de la verticalidad institucional (Saleebey, 2000).Siendo necesario que las personas diagnosticadas con un trastorno mental puedan compartir experiencias y espacios con otras personas con el objetivo de romper con las limitaciones y barreras personales, mediante la inversión en la comunidad (Brea y Gil, 2016). Esta práctica se basa en los principios y valores intrínsecos del trabajo social: el reconcomiendo de las capacidades, de las habilidades, y del conocimiento de las propias experiencias (Sebastin, 2014).Requiere también de una profunda implicación subjetiva en la aproximación a las personas, a sus limitaciones y/o a sus barreras mediante el reconociendo de su singularidad y dignidad, lo que implica el reconocimiento del otro como un individuo único y valioso para la sociedad en la que habita. Para lograr este propósito es necesario comprometer a los estamentos políticos en el mantenimiento de las personas en su comunidad, y transmitir el valor de la diversidad dentro de la misma. Olvidando viejos dogmas, en los que la suma de las demandas y situaciones desdibujaban a las personas solicitantes de atención (Breton, 1994; Lietz, 2007). 
Demanda también la interpelación a la comunidad para un cambio en la concepción de la responsabilidad de la adaptación a la comunidad como un hecho individual, para que pueda emerger la comprensión de las limitaciones y de las barreras como una cuestión política. Desde esta perspectiva se sustenta la idea de las personas con diversidad como una parte imprescindible y valiosa de la composición de la comunidad de referencia, manteniendo su capacidad en la toma de decisiones relacionadas con su vida. Se puede afirmar que la praxis del/la trabajador/a social para las personas diagnosticadas con un trastorno mental es central para la adquisición de confianza y en la autonomía sobre los propios procesos.

Es responsabilidad del trabajo social el reconocimiento de los conocimientos y de las experiencias vivenciales de las personas silenciadas por su diversidad, para lograr que la práctica del trabajo social devenga una plataforma que facilite la creación de nuevas instituciones de orden simbólico y formal, que amplíen el diálogo junto con los nuevos actores. La reflexión y el control en las actuaciones de las entidades políticas, y la ejecución de las directrices en salud mental se han de realizar desde el reconocimiento de la diversidad como un hecho enriquecedor para la sociedad de pertenencia.

\section{Referencias bibliográficas}

AlluÉ, M. (2003). Discapacitados. La reivindicación de la igualdad en la diferencia. Barcelona: Ediciones Bellaterra.

Asís, R. (2013). Sobre el modelo social de la discapacidad: Críticas y éxito. Papeles el tiempo de los derechos, 1, 1-12.

BANKS, S. (1997). Ética y valores en el Trabajo Social. Barcelona: Paidós.

BEECHER, B. (2009). The medical model, mental health practitioners, and individuals with schizophrenia and their families. Journal of Social Work Practice, 23(1), 9-20. http://dx.doi.org/10.1080/02650530902723282

BERGERON-LeCLERC, C. \& CORMIER, C. (2009). Les ingrédients magiques de la relation d'aide: Une exploration des facteurs à l'origine du succès de l'intervention dans le champ de la santé mentale. Service social 55 (1), 1-16. https://doi. org/10.7202/029486ar

BradHAW, W., ARmour, M.P. \& Roseborough, D. (2007). Finding a place in the world the experience of recovery from severe mental illness. Qualitative Social Work, 6(1), 27-47. https://doi.org/10.1177/1473325007074164

BREA, J. y GIL, H. (2016). Estigma y salud mental. Una reflexión desde el trabajo social. Trabajo Social Hoy, 78, 95-112. http://dx.doi.org/10.12960/ TSH.2016.0012 
BRETON, M. (1994). On the meaning of empowerment and empowerment-oriented social work. Social Work with Groups, 17(3), 23-27. http://dx.doi.org/10.1300/ J009v17n03_03

BUCK, J. (2013). Perceived stigma and societal discrimination in individuals with mental illness: Understanding the importance of a sense of community. Social Sciences Capstone Projects. Paper 22 http://commons.pacificu.edu/cgi/viewcontent. cgi?article $=1018$ E context $=$ cassoc

Carter, M.A., Pagliano, P.; Francis, A. \& Thorne, M. (2016). Australian University Students and Mental Health: Viewpoints from the literature. International Journal of Innovation, Creativity and Change, 3(3), 1-25 www. ijicc.net.

CASAl, J. C. (2010). Derechos y participación de las personas con diversidad mental. Cuadernos de Trabajo social, 23, 301-321.

CAzorla, J. (2014). Las relaciones sociales en Salud Mental: Estudio sobre la percepción del impacto de las relaciones sociales en la mejora de la autonomía de las personas con diversidad mental y la relación con el Trabajo Social. Alternativas. Cuadernos de Trabajo Social, 21,161-176. https://doi.org/10.14198/ ALTERN2014.21.08

Corrigan, P.W; River, L.P.; Lundin, R.L.; PenN, D.L.; UPhOFF-WasowsKi, K.; Campion, J.; Mathisen, J.; Gagnon, C.; Bergman.; Goldstein. \& Kubiak, M.A. (2001). Three strategies for changing attributions. Schizophrenia Bulletin, 27(2), 187-195. https://doi.org/10.1093/oxfordjournals.schbul.a006865

DERRIDA, J. (1997). El monolingüismo del otro, o la prótesis de origen. Buenos Aires: Manantial.

Dominelli, L. (1996). Deprofessionalizing Social Work: Anti-Oppressive Practice, Competencias and Postmodernism. British Journal of Social Work, 26(2), 153175. https://doi.org/10.1093/oxfordjournals.bjsw.a011077

Gelkorpf, M. \& RoE, D. (2014). Evaluating Outcome Domains Assessing Caregivers of Individuals with Mental Illes: A Review. Family process, 53(1), 150-174. https://doi.org/10.1111/famp.12056

Hyde, B., BOWLES, W. \& PAWAR, M. (2015). 'We're Still in There'-Consumer Voices on Mental Health Inpatient Care: Social Work Research Highlighting Lessons for Recovery Practice. British Journal of Social Work, 45(1), i62-i78. https:// doi.org/10.1093/bjsw/bcv093

IDARETA, F. (2013). E. Lévinas y el Trabajo Social: Más allá que de Jonia a Jena. Daímon. Revista Internacional de Filosofía, 58, 19-32.

IDARETA, F. (2014). Propuesta para la medición de la violencia al usuario en el trabajo social desde las perspectivas de E. Lévinas y J. Derrida. Portularia, XIV (1), 15-26. https://doi.org/10.5218/prts.2014.0002 
KARBAN, K. (2017). Developing a Health Inequalities Approach for Mental Health Social Work. British Journal of Social Work, 47 (3), 885-992. https://doi. org/10.1093/bjsw/bcw098

LiETZ, C.A. (2007). Strengths-Based Group Practice: Three Case Studies. Social Work with Groups, 30(2), 73-87. https://doi.org/10.1300/J009v30n02_07

Michaels, P.J.; LÓPEZ, M.; RÜSCH, N. \& CORRIGAN, P. W. (2012). Constructs and concepts comprising the stigma of mental illness. Psychology, Society $\varepsilon$ Education, 4(2), 183-194. https://doi.org/10.25115/psye.v4i2.490

Mulvale, G. \& BARTRAM, M. (2015). No More «Us» and «Them»: Integrating Recovery and Well-Being into a Conceptual Model for Mental Health Policy. Canadian Journal of Community Mental Health, 34(4), 31-67. https://doi. org/10.7870/cjcmh-2015-010

MuYor, J. (2011). La (con) ciencia del Trabajo Social en la discapacidad: Hacia un modelo de intervención social basado en derechos. Documentos de trabajo Social, 49, 9-33.

Palacios, A. y RomañaCH, J. (2006). El modelo de la diversidad: La bioética y los derechos humanos como herramientas para alcanzar la plena dignidad en la diversidad funcional. Madrid: Diversitas.

PAlacios, A. (2008). El modelo social de discapacidad: orígenes, caracterización y plasmación en la Convención Internacional sobre los Derechos de las Personas con Discapacidad. Madrid: Cinca.

Parra, B., Oriol, A. y Martínez, M. (2011): Trabajo social y enfermero en un equipo de atención precoz a la psicosis. Revista de Psicopatología y salud mental del niño y del adolescente, 18, 89-98.

PARAZElli, M. \& Bourbonnais, M. (2017). L'empowerment en travail social. Perspectives, enseignements et limites. Sciences et Actions Sociales, 6, 1-29.

SaleEbey, D. (2000). Power in the people: Strengths and hope. Advances in Social Work, 1(2), 127-136.

SEBASTIN, K.V. (2014). Tackling contemporary challenges throughs trengths based approach in Social Work. International Journal of Social Work and Human Services Practice, 2(6), 291-295. DOI: 10.13189/ijrh.2014.020613

SEN, A. (1988). ¿Igualdad de qué? en Sterling M. McMurrin (edit). Libertad, igualdad y derecho. Barcelona: Ariel.

SCHÖN, U.K., DENHOV, A. \& TOPOR, A. (2009). Social relationships as a decisive factor in recovering from severe mental illness. International Journal of Social Psychiatry, 55(4), 336-347. https://doi.org/10.1177/0020764008093686

STARnino, V.R. (2009). An Integral Approach to Mental Health Recovery: Implications for Social Work. Journal of Human Behavior in the Social Environment, 19, 820-842. https://doi.org/10.1080/10911350902988019

Tew, J., Shula, R., Slade, M., Bird, V., Melton, J \& Le Boutillier, Cl. (2012). Social Factors and Recovery from Mental Health Difficulties: A Review of 
the Evidence. British Journal of Social Work, 42 (3), 443-460. https://doi. org/10.1093/bjsw/bcr076

Toboso, M. y ARnau, M.S. (2008). La discapacidad dentro del enfoque de capacidad y funcionamiento de Amartya Sen. Araucaria Revista Iberoamericana de Filosofía, Política y Humanidades, 20, 64-94. <http://www.redalyc.org/articulo. oa? id=28212043004 > ISSN 1575-6823.

Victoria, J. A. (2013). El modelo social de la discapacidad: una cuestión de derechos humanos. Boletín Mexicano de Derecho Comparado, 46 (138), 1093-1109. https://doi.org/10.1016/S0041-8633(13)71162-1

Velarde, V. (2012). Los modelos de la discapacidad: un recorrido histórico. Revista empresa y humanidades, 1, 115-136.

Williams, CH., Almeida, M. \& Knyahnytska, Y. (2015). Towards a Biopsychosociopolitical Frame for Recovery in the Context of Mental Illness. British Journal of Social Work, 45 (1), i9-i26. https://doi.org/10.1093/bjsw/ bcv100 